\title{
On the symmetry of four particles in a one-dimensional box with harmonic interaction
}

\author{
Francisco M. Fernández * \\ INIFTA (CONICET, UNLP), División Química Teórica \\ Blvd. 113 S/N, Sucursal 4, Casilla de Correo 16, 1900 La Plata, Argentina
}

\begin{abstract}
We show that a system of four particles in a one-dimensional box with a two-particle harmonic interaction can by described by means of the symmetry point group $O_{h}$. Group theory proves useful for the discussion of both the small-box and large-box regimes. We apply perturbation theory and obtain the corrections of first order for the lowest states. We carry out a simple Rayleigh-Ritz variational calculation with basis sets adapted to the symmetries of the system. We also obtain alternative variational results for the first three lowest energy levels that are more suitable for larger box sizes.
\end{abstract}

\section{Introduction}

During the last decades there has been great interest in the model of a harmonic oscillator confined to boxes of different shapes, sizes and dimensions. Such model has been suitable for the study of several physical problems ranging from dynamical friction in star clusters to magnetic properties of solids and impurities in quantum dots (see [1,2] for a review of the relevant literature on the subject). In addition to it, systems of few identical particles in one dimension

*E-mail: fernande@quimica.unlp.edu.ar 
have proved to exhibit a rich phenomenological structure resembling that of realistic systems [3 6]. In two recent papers Amore and Fernández discussed the problems posed by two [1] and three [2] particles confined in a one-dimensional box with impenetrable walls that interact through harmonic forces. They found that a straightforward application of group theory considerably facilitates the analysis of the solutions to the Schrödinger equation. In particular, an accurate Rayleigh-Ritz variational calculation revealed that the energies of the threeparticle model as functions of the box length exhibit a most interesting pattern of avoided crossings between pairs of states of the same symmetry [2].

The purpose of this paper is to discuss the case of four particles in a one dimensional box that also interact through harmonic forces. The reason for choosing such interaction is that it has proved quite useful in the past (see the references in [2]) and because the calculation of the matrix elements of the resulting potential is quite simple. In section 2 we introduce the model, section 3 shows a perturbation approach to the small-box regime and a Rayleigh-Ritz calculation with symmetry-adapted basis sets, section 4 describes the largebox limit, in section 5 we discuss a simple variational calculation that is more suitable for larger box sizes and in section [ we draw conclusions. There is also an Appendix outlining the construction of the projection operators used in all the calculations just described.

\section{Four particles in a one-dimensional box}

We first consider $N$ interacting point particles of mass $m$ in a one-dimensional box of length $L=2 a$ with Hamiltonian

$$
H=-\frac{\hbar^{2}}{2 m} \sum_{i=1}^{N} \frac{\partial^{2}}{\partial x_{i}^{2}}+\sum_{i=1}^{N-1} \sum_{j=i+1}^{N} W\left(\left|x_{i}-x_{j}\right|\right),
$$

where $x_{i}$ is the coorinate of the $i$-th particle. The boundary conditions are determined by the impenetrable walls of the box

$$
\Psi\left(x_{1}, x_{2}, \ldots, x_{i}= \pm a, \ldots, x_{N}\right)=0, i=1,2, \ldots, N .
$$


The Hamiltonian operator is invariant under the $N$ ! permutations of the particle coordinates as well as under parity inversion $\mathbf{x} \rightarrow-\mathbf{x}$. The $2 N ! N \times N$ matrices that produce all the permutations of the sets $\left\{x_{1}, x_{2}, \ldots, x_{N}\right\}$ and $\left\{-x_{1},-x_{2}, \ldots,-x_{N}\right\}$ form a group given by the product $S_{N} \otimes O(1)[7,8]$. When $N=2$ the group is also called $C_{2 h}$ (in principle we can also use $D_{2}$ or $C_{2 v}$ that are isomorphic to $C_{2 h}$ ) and when $N=3$ we can resort to either $D_{3 d}$ or $D_{3 h}[9]$. Both cases have already been treated by group theory in earlier papers 1,2,

In order to solve the Schrödinger equation it is convenient to define the dimensionless particle coordinates $q_{i}=x_{i} / a$ and the dimensionless Hamiltonian

$$
H_{d}=\frac{2 m a^{2}}{\hbar^{2}} H=-\sum_{i=1}^{N} \frac{\partial^{2}}{\partial q_{i}^{2}}+\lambda \sum_{i=1}^{N-1} \sum_{j=i+1}^{N} w\left(\left|q_{i}-q_{j}\right|\right),
$$

where $\lambda w\left(\left|q_{i}-q_{j}\right|\right)=2 m a^{2} W\left(a\left|q_{i}-q_{j}\right|\right) / \hbar^{2}$. The boundary conditions for the eigenfunctions $\psi$ of this operator now become

$$
\psi\left(q_{1}, q_{2}, \ldots, q_{i}= \pm 1, \ldots, q_{N}\right)=0, i=1,2, \ldots, N .
$$

From now on we only consider the dimensionless Hamiltonian (3) and omit the subscript $d$. In order to facilitate the numerical calculations we choose a harmonic interaction of the form $W\left(\left|x_{i}-x_{j}\right|\right)=\frac{k}{2}\left(x_{i}-x_{j}\right)^{2}$ that leads to $\lambda=$ $m a^{2} k / \hbar^{2}$ and $w\left(\left|q_{i}-q_{j}\right|\right)=\left(q_{i}-q_{j}\right)^{2}$.

For $N=4$ our problem just reduces to solving the Schrödinger equation for the operator

$$
\begin{aligned}
H=-\quad & \left(\frac{\partial^{2}}{\partial q_{1}^{2}}+\frac{\partial^{2}}{\partial q_{2}^{2}}+\frac{\partial^{2}}{\partial q_{3}^{2}}+\frac{\partial^{2}}{\partial q_{4}^{2}}\right)+ \\
+ & \lambda\left[\left(q_{1}-q_{2}\right)^{2}+\left(q_{1}-q_{3}\right)^{2}+\left(q_{1}-q_{4}\right)^{2}+\left(q_{2}-q_{3}\right)^{2}\right. \\
+ & \left.\left(q_{2}-q_{4}\right)^{2}+\left(q_{3}-q_{4}\right)^{2}\right]
\end{aligned}
$$

with the boundary conditions

$$
\psi\left( \pm 1, q_{2}, q_{3}, q_{4}\right)=\psi\left(q_{1}, \pm 1, q_{3}, q_{4}\right)=\psi\left(q_{1}, q_{2}, \pm 1, q_{4}\right)=\psi\left(q_{1}, q_{2}, q_{3}, \pm 1\right)=0
$$

In this case the group $S_{4} \otimes O(1)$ is isomorphic to $O_{h}$ and in this paper we resort to the character table of the latter group [9]. 


\section{Small-box-regime}

When $\lambda$ is sufficiently small (sufficiently small box size $L$ ) we can estimate the energy levels by means of perturbation theory. The Schrödinger equation is exactly solvable when $\lambda=0$ and the eigenvalues and eigenfunctions of $H_{0}=$ $H(\lambda=0)$ are

$$
\begin{aligned}
E_{n_{1} n_{2} n_{3} n_{4}}^{(0)} & =\frac{\pi^{2}}{4}\left(n_{1}^{2}+n_{2}^{2}+n_{3}^{2}+n_{4}^{2}\right), n_{1}, n_{2}, n_{3}, n_{4}=1,2, \ldots \\
\psi_{n_{1} n_{2} n_{3} n_{4}}^{(0)}\left(q_{1}, q_{2}, q_{3}, q_{4}\right) & =\phi_{n_{1}}\left(q_{1}\right) \phi_{n_{2}}\left(q_{2}\right) \phi_{n_{3}}\left(q_{3}\right) \phi_{n_{4}}\left(q_{4}\right) \\
\phi_{n}(q) & =\sin \frac{n \pi(q+1)}{2}
\end{aligned}
$$

Note that $\phi_{n}(-q)=(-1)^{n-1} \phi_{n}(q)$.

In order to facilitate the discussion of the results we introduce the notation $\{a, b, c, d\}_{P}$ to indicate the set of all distinct permutations of four elements that may be either coordinates or quantum numbers. For example, each 4tuple of quantum numbers in the set $\left\{n_{1}, n_{2}, n_{3}, n_{4}\right\}_{P}$ leads to the same unperturbed energy $E_{n_{1} n_{2} n_{3} n_{4}}^{(0)}$. We may eventually add accidental Pythagorean degeneracies of the form $m_{1}^{2}+m_{2}^{2}+m_{3}^{2}+m_{4}^{2}=n_{1}^{2}+n_{2}^{2}+n_{3}^{2}+n_{4}^{2}$, where $\left(m_{1}, m_{2}, m_{3}, m_{4}\right) \notin\left\{n_{1}, n_{2}, n_{3}, n_{4}\right\}_{P}$ [10]. If such energy level is $g$-fold degenerate then the perturbation corrections of first order to the eigenfunctions will be of the form

$$
\psi^{(1)}=\sum_{j=1}^{g} c_{j}^{(1)} \psi_{j}^{(0)},
$$

where $j$ denotes a 4 -tuple $\left(n_{1}, n_{2}, n_{3}, n_{4}\right)$. The coefficients $c_{j}^{(1)}$ are solutions to the secular equation

$$
\left(\mathbf{H}^{\prime}-E^{(1)} \mathbf{I}\right) \mathbf{c}^{(1)}=0
$$

where $\mathbf{H}^{\prime}$ is the $g \times g$ matrix of the perturbation $H^{\prime}=H-H_{0}$ in the set of degenerate eigenfunctions $\left\{\psi_{j}^{(0)}, j=1,2, \ldots, g\right\}$, I is the $g \times g$ identity matrix, $\mathbf{c}^{(1)}$ is a column vector with elements $c_{j}^{(1)}$ and $E^{(1)}$ is one of the $g$ roots of the secular determinant $\left|\mathbf{H}^{\prime}-E^{(1)} \mathbf{I}\right|=0$.

In order to determine the symmetry of the solution $\psi^{(1)}$, which provides a suitable label for the corresponding root $E^{(1)}$, we apply the projection operators 
$P_{S}$ associated to the irreducible representations (irreps) $S$ of the group $O_{h}[9$. The result is well known to be $P_{S} \psi^{(1)}=\psi^{(1)}$ if $\psi^{(1)}$ is a basis for the irrep $S$ or $P_{S} \psi^{(1)}=0$ otherwise. The construction of the projection operators is outlined in the Appendix. In this way we obtain the following results for the first energy levels:

$$
\begin{aligned}
& E_{1 A_{1 g}}=\pi^{2}+\frac{4\left(\pi^{2}-6\right)}{\pi^{2}} \lambda+\ldots, \\
& E_{1 A_{1 u}}=\frac{7 \pi^{2}}{4}+\frac{216 \pi^{4}-1053 \pi^{2}-4096}{54 \pi^{4}} \lambda+\ldots, \\
& E_{1 T_{2 u}}=\frac{7 \pi^{2}}{4}+\frac{648 \pi^{4}-3159 \pi^{2}+4096}{162 \pi^{4}} \lambda+\ldots, \\
& E_{2 A_{1 g}}=\frac{5 \pi^{2}}{2}+\frac{324 \pi^{4}-1215 \pi^{2}-8192}{81 \pi^{4}} \lambda+\ldots, \\
& E_{1 T_{2 g}}=\frac{5 \pi^{2}}{2}+\frac{4 \pi^{2}-15}{\pi^{2}} \lambda+\ldots, \\
& E_{1 E_{g}}=\frac{5 \pi^{2}}{2}+\frac{324 \pi^{4}-1215 \pi^{2}+4096}{81 \pi^{4}} \lambda+\ldots, \\
& E_{3 A_{1 g}}=3 \pi^{2}+\frac{4\left(3 \pi^{2}-14\right)}{3 \pi^{2}} \lambda+\ldots, \\
& E_{2 T_{2 g}}=3 \pi^{2}+\frac{4\left(3 \pi^{2}-14\right)}{3 \pi^{2}} \lambda+\ldots, \\
& E_{2 A_{1 u}}=\frac{13 \pi^{2}}{4}+\frac{216 \pi^{4}-567 \pi^{2}-4096}{54 \pi^{4}} \lambda+\ldots, \\
& E_{2 T_{2 u}}=\frac{13 \pi^{2}}{4}+\frac{648 \pi^{4}-1701 \pi^{2}+4096}{162 \pi^{4}} \lambda+\ldots, \\
& E_{3 A_{1 u}}=\frac{15 \pi^{2}}{4}+\frac{405000 \pi^{4}-1434375 \pi^{2}-8105984}{101250 \pi^{4}} \lambda+\ldots, \\
& E_{3 T_{2 u}}=\frac{15 \pi^{2}}{4}+\frac{405000 \pi^{4}-1434375 \pi^{2}-6144 \sqrt{424321}-1280000}{101250 \pi^{4}} \lambda+\ldots, \\
& E_{1 E_{u}}=\frac{15 \pi^{2}}{4}+\frac{405000 \pi^{4}-1434375 \pi^{2}-425984}{101250 \pi^{4}} \lambda+\ldots,
\end{aligned}
$$




$$
\begin{aligned}
E_{4 T_{2 u}}= & \frac{15 \pi^{2}}{4}+\frac{405000 \pi^{4}-1434375 \pi^{2}+6144 \sqrt{424321}-1280000}{101250 \pi^{4}} \lambda+\ldots, \\
E_{1 T_{1 u}}= & \frac{15 \pi^{2}}{4}+\frac{405000 \pi^{4}-1434375 \pi^{2}+5545984}{101250 \pi^{4}} \lambda+\ldots, \\
E_{4 A_{1 g}}=4 \pi^{2}+\frac{2\left(2 \pi^{2}-3\right)}{\pi^{2}} \lambda+\ldots, & \\
E_{5 A_{1 g}}= & \frac{9 \pi^{2}}{2}+\frac{202500 \pi^{4}-489375 \pi^{2}-5545984}{50625 \pi^{4}} \lambda+\ldots, \\
E_{3 T_{2 g}}= & \frac{9 \pi^{2}}{2}+\frac{202500 \pi^{4}-489375 \pi^{2}-6144 \sqrt{53329}-1386496}{50625 \pi^{4}} \lambda+\ldots, \\
E_{4 T_{2 g}}= & \frac{9 \pi^{2}}{2}+\frac{202500 \pi^{4}-489375 \pi^{2}+6144 \sqrt{53329}-1386496}{50625 \pi^{4}} \lambda+\ldots, \\
E_{1 T_{1 g}} & =\frac{9 \pi^{2}}{2}+\frac{202500 \pi^{4}-489375 \pi^{2}+2772992}{50625 \pi^{4}} \lambda+\ldots, \\
E_{2 E_{g}} & =\frac{9 \pi^{2}}{2}+\frac{202500 \pi^{4}-489375 \pi^{2}+2772992}{50625 \pi^{4}} \lambda+\ldots,
\end{aligned}
$$

where $A_{1 g}, A_{2 g}, A_{1 u}$, and $A_{2 u}$ are nondegenerate, $E_{g}$ and $E_{u}$ are two-fold degenerate, and $T_{1 g}, T_{2 g}, T_{1 u}$ and $T_{2 u}$ are three-fold degenerate.

We appreciate that the degeneracy of the unperturbed states is partially removed by the perturbation. The remaining degeneracies are expected to be broken at higher perturbation orders. One does not expect that such remaining degeneracies are due to an unknown hidden symmetry [10] because they are rather inconsistent. For example, in the case $\{1,1,1,3\}_{P}$ we have $E_{3 A_{1 g}}=E_{2 T_{2 g}}$; however, $\{1,2,2,3\}_{P}$ leads to $E_{5 A_{1 g}}<E_{3 T_{2 g}}<E_{4 T_{2 g}}<E_{2 E_{g}}=E_{1 T_{1 g}}$ where the state $A_{1 g}$ and the three states $T_{2 g}$ are not degenerate as in the preceding case.

Figure 1 shows the lowest energy levels in the interval $0 \leq \lambda \leq 1$ where perturbation theory is expected to yield sufficiently accurate results. We also carried out a simple Rayleigh-Ritz variational calculation with symmetry-adapted basis sets for $A_{1 g}$ and $T_{2 g}$ choosing only those functions coming from $\{1,1,1,1\}$, $\{1,1,2,2\}_{P}$ and $\{1,1,1,3\}_{P}$ in order to show the splitting of the levels $E_{3 A_{1 g}}$ and $E_{2 T_{2 g}}$. These levels appear to be degenerate in the upper subfigure but the finer scale of the lower one clearly reveals the splitting that takes place at the 
second order of perturbation theory $\left(E_{3 A_{1 g}}>E_{2 T_{2 g}}\right)$. The symmetry-adapted basis set was constructed by straightforward application of the projection operators $P_{A_{1 g}}$ and $P_{T_{2 g}}$ to the zeroth-order eigenfunctions (7) (see [1,2] for more details).

\section{Large-box-regime}

When $L \rightarrow \infty(\lambda \rightarrow \infty)$ we have a system of four unconfined particles with harmonic-pair interaction. In order to discuss this case it is convenient to define the new coordinates

$$
\begin{aligned}
& \xi_{1}=\frac{\sqrt{2} q_{2}}{2}-\frac{\sqrt{2} q_{1}}{2} \\
& \xi_{2}=-\frac{\sqrt{6} q_{1}}{6}-\frac{\sqrt{6} q_{2}}{6}+\frac{\sqrt{6} q_{3}}{3} \\
& \xi_{3}=-\frac{\sqrt{3} q_{1}}{6}-\frac{\sqrt{3} q_{2}}{6}-\frac{\sqrt{3} q_{3}}{6}+\frac{\sqrt{3} q_{4}}{2} \\
& \xi_{4}=\frac{q_{1}+q_{2}+q_{3}+q_{4}}{2}
\end{aligned}
$$

because the Hamiltonian operator becomes

$$
H=-\left(\frac{\partial^{2}}{\partial \xi_{1}^{2}}+\frac{\partial^{2}}{\partial \xi_{2}^{2}}+\frac{\partial^{2}}{\partial \xi_{3}^{2}}+\frac{\partial^{2}}{\partial \xi_{4}^{2}}\right)+4 \lambda\left(\xi_{1}^{2}+\xi_{2}^{2}+\xi_{3}^{2}\right)
$$

We appreciate that the center of mass described by the coordinate $\xi_{4}$ moves freely while there is harmonic oscillation along the 3 remaining coordinates. The eigenvalues and eigenfunctions are expected to be

$$
\begin{aligned}
E_{K, n_{1}, n_{2}, n_{3}} & =K^{2}+2 \sqrt{\lambda}\left(2 n_{1}+2 n_{2}+2 n_{3}+3\right), \\
\psi_{K, n_{1}, n_{2}, n_{3}}\left(\xi_{1}, \xi_{2}, \xi_{3}, \xi_{4}\right) & =\exp \left(i K \xi_{4}\right) \chi_{n_{1}}\left(\xi_{1}\right) \chi_{n_{2}}\left(\xi_{2}\right) \chi_{n_{3}}\left(\xi_{3}\right), \\
n_{1}, n_{2}, n_{3} & =0,1, \ldots,-\infty<K<\infty,
\end{aligned}
$$

where $\chi_{n}(\xi)$ is a harmonic-oscillator eigenfunction.

The connection between the small and large box regimes is given by

$$
\lim _{\lambda \rightarrow \infty} \lambda^{-1 / 2} E_{m_{1}, m_{2} m_{3}, m_{4}}(\lambda)=2\left(2 n_{1}+2 n_{2}+2 n_{3}+3\right) .
$$


When carrying out this limit we should take into account that the symmetry of the eigenfunction should be conserved as $\lambda \rightarrow \infty$ and that $\exp \left(i K \xi_{4}\right)$ does not exhibit a definite symmetry. Therefore, in order to make a connection between both regimes the eigenfunctions in the large-box case should be of the form

$$
\begin{aligned}
& \psi_{K, n_{1}, n_{2}, n_{3}}^{c}\left(\xi_{1}, \xi_{2}, \xi_{3}, \xi_{4}\right)=\cos \left(K \xi_{4}\right) \chi_{n_{1}}\left(\xi_{1}\right) \chi_{n_{2}}\left(\xi_{2}\right) \chi_{n_{3}}\left(\xi_{3}\right), \\
& \psi_{K, n_{1}, n_{2}, n_{3}}^{s}\left(\xi_{1}, \xi_{2}, \xi_{3}, \xi_{4}\right)=\sin \left(K \xi_{4}\right) \chi_{n_{1}}\left(\xi_{1}\right) \chi_{n_{2}}\left(\xi_{2}\right) \chi_{n_{3}}\left(\xi_{3}\right) .
\end{aligned}
$$

In order to determine the symmetry of a given eigenfunction in the largebox limit we take into account that $\xi_{4}$ is basis for the irrep $A_{1 u}$ while the set $\left\{\xi_{1}, \xi_{2}, \xi_{3}\right\}$ is a basis for the irrep $T_{2 u}$. Therefore, $\cos \left(K \xi_{4}\right)$ and $\sin \left(K \xi_{4}\right)$ are basis for the irreps $A_{1 g}$ and $A_{1 u}$, respectively. By means of the direct product of irreps we obtain the symmetry of any eigenfunction of the form (22). For example, when $\left\{n_{1}, n_{2}, n_{3}\right\}_{P}=\{0,0,1\}_{P}$ the three possible products $\chi_{n_{1}}\left(\xi_{1}\right) \chi_{n_{2}}\left(\xi_{2}\right) \chi_{n_{3}}\left(\xi_{3}\right)$ are basis for $T_{2 u}$ and the resulting functions $\psi_{K, n_{1}, n_{2}, n_{3}}^{c}\left(\xi_{1}, \xi_{2}, \xi_{3}, \xi_{4}\right)$ and $\psi_{K, n_{1}, n_{2}, n_{3}}^{s}\left(\xi_{1}, \xi_{2}, \xi_{3}, \xi_{4}\right)$ are basis for $T_{2 u}$ and $T_{2 g}$, respectively.

\section{Simple variational method}

In order to obtain accurate variational results for large values of $\lambda$ as was done in the case of two particles [1] we should try variational functions of the form

$$
F\left(q_{1}, q_{2}, q_{3}, q_{4}\right)=G\left(q_{1}, q_{2}, q_{3}, q_{4}\right) \exp \left[-a\left(\xi_{1}^{2}+\xi_{2}^{2}+\xi_{3}^{2}\right)\right]
$$

where $G\left(q_{1}, q_{2}, q_{3}, q_{4}\right)$ satisfies the boundary conditions at the box walls and $\exp \left[-a\left(\xi_{1}^{2}+\xi_{2}^{2}+\xi_{3}^{2}\right)\right]$ provides the correct asymptotic behaviour of the wavefunction of the free oscillator. The variational parameter $a$ will increase from $a_{0}$ to infinity as $\lambda$ increases from zero to infinity.

However, since this calculation is rather cumbersome here we try a much simpler one with a variational function of the form

$$
F\left(q_{1}, q_{2}, q_{3}, q_{4}\right)=G\left(q_{1}, q_{2}, q_{3}, q_{4}\right) \exp \left[-a\left(q_{1}^{2}+q_{2}^{2}+q_{3}^{2}+q_{4}^{2}\right)\right]
$$


where we clearly sacrifice the correct description of the asymptotic behaviour when $\lambda \rightarrow \infty$. For example,

$$
\begin{aligned}
F_{A_{1 g}}\left(q_{1}, q_{2}, q_{3}, q_{4}\right)= & N\left(q_{1}^{2}-1\right)\left(q_{2}^{2}-1\right)\left(q_{3}^{2}-1\right)\left(q_{4}^{2}-1\right) \times \\
& \times \exp \left[-a\left(q_{1}^{2}+q_{2}^{2}+q_{3}^{2}+q_{4}^{2}\right)\right], \\
F_{A_{1 u}}\left(q_{1}, q_{2}, q_{3}, q_{4}\right)= & N \xi_{4}\left(q_{1}^{2}-1\right)\left(q_{2}^{2}-1\right)\left(q_{3}^{2}-1\right)\left(q_{4}^{2}-1\right) \times \\
& \times \exp \left[-a\left(q_{1}^{2}+q_{2}^{2}+q_{3}^{2}+q_{4}^{2}\right)\right],
\end{aligned}
$$

and

$$
\begin{aligned}
F_{T_{2 u}}\left(q_{1}, q_{2}, q_{3}, q_{4}\right)= & N\left(q_{1}^{2}-1\right)\left(q_{2}^{2}-1\right)\left(q_{3}^{2}-1\right)\left(q_{4}^{2}-1\right) \times \\
& \times \exp \left[-a\left(q_{1}^{2}+q_{2}^{2}+q_{3}^{2}+q_{4}^{2}\right)\right]\left\{\begin{array}{l}
\xi_{1} \\
\xi_{2} \\
\xi_{3}
\end{array}\right.
\end{aligned}
$$

are expected to yield approximations to the first energy levels $E_{1 A_{1 g}}<E_{1 A_{1 u}}<$ $E_{1 T_{2 u}}$.

Figure 2 shows the perturbation and variational results for those three states. Both the perturbation corrections of first order and the variational approaches are upper bounds to the corresponding energies because the variational principle applies to the lowest state of each symmetry. Since the perturbation expressions shown in section 3 ( $E_{P T}$ from now on) yield the exact result when $\lambda=0$ they are expected to be more accurate than the variational results $\left(E_{v a r}\right)$ for sufficiently small $\lambda$. Figure 2 reveals that $E_{\text {var }}\left\langle E_{P T}\right.$ for $\lambda>\lambda_{c}$ that tells us that $E_{v a r}$ is more accurate for sufficiently large values of $\lambda$. As expected, the perturbation expressions exhibit a wrong behaviour for large values of $\lambda$. On the other hand, $E_{\text {var }}$ increases correctly as $\sqrt{\lambda}$ but the choice of an incorrect exponential factor in the trial function leads to a wrong coefficient of the leading term of the energy; for example a numerical calculation suggests that

$$
\lim _{\lambda \rightarrow \infty} \lambda^{-1 / 2} E_{1 A_{1 g}}=\sqrt{48}>6 .
$$




\section{Conclusions}

Throughout this paper, as well as in the two earlier ones 1,2], we have shown that group theory is useful for the analysis of systems of particles in a onedimensional box. In the present case we can label the states of the system of four particles by means of the irreps of the point group $O_{h}$. The knowledge of the symmetry of the states for finite $\lambda$ and for $\lambda \rightarrow \infty$ facilitates the analysis of the connection between the states of the confined and free systems, respectively. In addition to it, point group proves suitable for the construction of simple variational trial functions like those in section 5 as well as for the construction of symmetry-adapted basis sets for more accurate calculations like the RayleighRitz method used in section 3 .

\section{References}

[1] P. Amore and F. M. Fernández, Two-particle harmonic oscillator in a onedimensional box, Acta Polytech. 50 (2010) 17-24. arXiv:0908.0006 [quant$\mathrm{ph}]$.

[2] P. Amore and F. M. Fernández, On the symmetry of three identical interacting particles in a one-dimensional box, Ann. Phys. 362 (2015) 118-129.

[3] N. L. Harshman, Symmetries of three harmonically trapped particles in one dimension, Phys. Rev. A 86 (2012) 052122.

[4] N. L. Harshman, Spectroscopy for a few atoms harmonically trapped in one dimension, Phys. Rev. A 89 (2014) 033633.

[5] N. L. Harshman, One-dimensional traps, two-body interactions, few-body symmetries: I. One, two, and three particles, Few-Body Syst. 57 (2016) $11-43$.

[6] N. L. Harshman, One-dimensional traps, two-body interactions, few-body symmetries. II. N particles, Few-Body Syst. 57 (2016) 45-69. 
[7] M. Hammermesh, Group Theory and its Application to Physical Problems, Addison-Wesley, Reading, Massachussets, 1962).

[8] M Tinkham, Group Theory and Quantum Mechanics, McGraw-Hill, New York, 1964).

[9] F. A. Cotton, Chemical Applications of Group Theory, Third (John Wiley \& Sons, New York, 1990).

[10] F. M. Fernández, On the symmetry of the quantum-mechanical particle in a cubic box, 2013. arXiv:1310.5136 [quant-ph].

\section{Appendix: Construction of projection operators}

In this appendix we outline the procedure for the construction of the projection operators that enabled us to determine the symmetry of the corrections of first order to the eigenfunctions as well as to construct symmetry-adapted basis sets and the variational trial functions.

First, we build a set of 48 matrices $G_{M}=\left\{\mathbf{M}_{j}, j=1,2, \ldots, 48\right\}$ given by the 24 permutations of the rows of the $4 \times 4$ identity matrix $\mathbf{I}$ and the 24 permutations of the rows of $\mathbf{- I}$. This set of matrices is a group with respect to the matrix product $\mathbf{M}_{i} \cdot \mathbf{M}_{j}$. Second, we define a set $G_{O}=\left\{O_{j}, j=1,2, \ldots, 48\right\}$ of linear invertible operators according to the rule

$$
O_{j} f(\mathbf{q})=f\left(\mathbf{M}_{j}^{-1} \mathbf{q}\right)
$$

where $\mathbf{q}$ is a column vector with elements $q_{i}$ (the four dimensionless particle coordinates) and $f(\mathbf{q})$ is an arbitrary function of them. These operators form a group with respect to the composition $O_{i} \circ O_{j}$. If $\mathbf{M}_{i}$ and $\mathbf{M}_{j}$ are the matrix representations of $O_{i}$ and $O_{j}$, respectively, then $\mathbf{M}_{i} \cdot \mathbf{M}_{j}$ is the matrix representation of $O_{i} \circ O_{j}$. In other words, the groups $G_{M}$ and $G_{O}$ are isomorphic.

Third, we determine the classes for the group of matrices $G_{M}$ in the usual way. Two matrices $\mathbf{M}_{i}$ and $\mathbf{M}_{j}$ belong to the same class if $\mathbf{M}_{k} \cdot \mathbf{M}_{i} \cdot \mathbf{M}_{k}^{-1}=\mathbf{M}_{j}$ for some $\mathbf{M}_{k} \in G_{M}$. Fourth, we calculate the traces, determinants and orders 
of the matrices in every class. In this way we connect each class of matrices $\mathbf{M}_{j}$ (or operators $O_{j}$ ) with the corresponding class of symmetry operations that appear in the character table of the group $O_{h}[9]$.

Finally, we obtain the projection operators by means of the well known expression [7- 9 ]

$$
P_{S}=\frac{n_{S}}{h} \sum_{j=1}^{h} \chi_{j}(S) O_{j},
$$

where $h=48$ is the order of the group, $n_{S}$ is the dimension of the irreducible representation $S$ and $\chi_{j}(S)$ is the character of the operation $O_{j}$ for $S$ that appears in the character table. Since the matrices in a class share the same trace it is sufficient to obtain a one-to-one correspondence between the classes of matrices and the classes of symmetry operations.

The application of the projection operators to the eigenfunctions of order zero given by some of the sets of quantum numbers $\left\{n_{1}, n_{2}, n_{3}, n_{4}\right\}_{P}$ yields the following irreps:

\begin{tabular}{|l|c|c|l|} 
Quantum numbers & Number of states & $E_{0}$ & Symmetry \\
\hline$\{1,1,1,1\}$ & 1 & $\pi^{2}$ & $1 A_{1 g}$ \\
$\{1,1,1,2\}_{P}$ & 3 & $7 \pi^{2} / 4$ & $1 A_{1 u}, 1 T_{2 u}$ \\
$\{1,1,2,2\}_{P}$ & 6 & $5 \pi^{2} / 2$ & $2 A_{1 g}, 1 T_{2 g}, 1 E_{g}$ \\
$\{1,1,1,3\}_{P}$ & 4 & $3 \pi^{2}$ & $3 A_{1 g}, 2 T_{2 g}$ \\
$\{1,2,2,2\}_{P}$ & 4 & $13 \pi^{2} / 4$ & $2 A_{1 u}, 2 T_{2 u}$ \\
$\{1,1,2,3\}_{P}$ & 12 & $15 \pi^{2} / 4$ & $3 A_{1 u}, 3 T_{2 u}, 1 E_{u}, 4 T_{2 u}, 1 T_{1 u}$ \\
$\{2,2,2,2\}_{P}$ & 1 & $4 \pi^{2}$ & $4 A_{1 g}$ \\
$\{1,2,2,3\}_{P}$ & 12 & $9 \pi^{2} / 2$ & $5 A_{1 g}, 3 T_{2 g}, 4 T_{2 g}, 2 E_{g}, 1 T_{1 g}$ \\
$\{1,1,1,4\}_{P}$ & 4 & $19 \pi^{2} / 4$ & $4 A_{1 u}, 5 T_{2 u}$ \\
$\{1,1,3,3\}_{P}$ & 6 & $5 \pi^{2}$ & $6 A_{1 g}, 3 E_{g}, 5 T_{2 g}$ \\
$\{2,2,2,3\}_{P}$ & 4 & $21 \pi^{2} / 4$ & $5 A_{1 u}, 6 T_{2 u}$ \\
$\{1,1,2,4\}_{P}$ & 12 & $11 \pi^{2} / 2$ & $7 A_{1 g}, 2 T_{1 g}, 6 T_{2 g}, 4 E_{g}, 2 T_{1 g}$
\end{tabular}



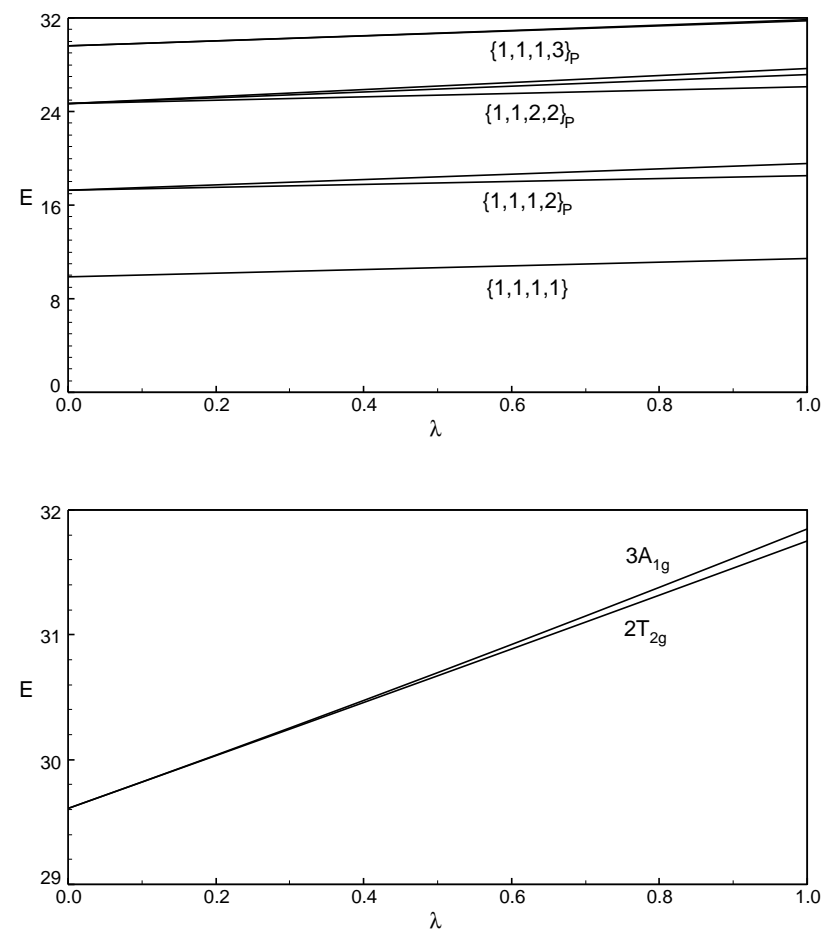

Figure 1: Lowest eigenvalues calculated by means of perturbation theory and the Raleigh-Ritz variational method

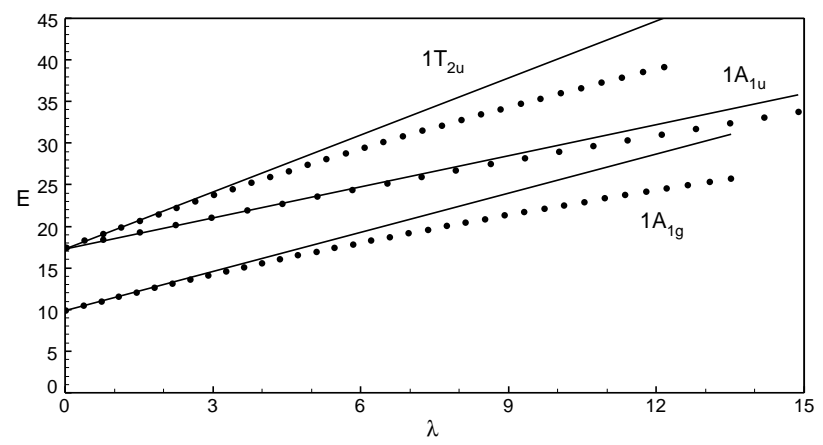

Figure 2: Lowest three eigenvalues calculated by means of perturbation theory (solid line) and the variational method (circles) 